\title{
The use of modern technologies based on telemedicine in wound care: experience in high-tyrrhenian region and the province of Cosenza, Italy
}

\author{
Francesco Giacinto, ${ }^{1}$ Ciro Falasconi, ${ }^{2}$ Elisabetta Giacinto, ${ }^{1}$ Manuela Germano, ${ }^{1}$ Domenica Ciuffoletti ${ }^{1}$ \\ ${ }^{1}$ Ambulatorio di Vulnologia, CAPT di Praia a Mare, ASP Cosenza; ${ }^{2}$ Ambulatorio per la Cura delle Ulcere Cutanee, ASL Napoli 1 \\ Centro, Napoli, Italy
}

\begin{abstract}
Chronic skin wounds (CSW) are a chronic illness that affects a large part of people forced to bed or home for disabling diseases; the chapter of cure and care of patients affected by this pathology represents an important commitment in terms of human and economic resources for the health system and, if not correctly managed, could identify with a threat to the sustainability of the system itself in Italy. The authors present the results of an experimentation carried out using the technologies and tools of Telemedicine with the aim of highlighting the problems and needs of home care of CSW. A telemedicine project was launched in which patients affected by CSW were followed remotely, directly from their home, using tablets and a program (application, APP) for the management of images of the CSW and of the clinical data. Results were compared, in terms of expenditure (number of home medical visits, mileage reimbursements) obtained in the treatment of patients with CSW in two semesters: the first (from October 2016 to April 2016) in which patients were followed in the traditional way and the second (from October 2016 to April 2017) in which the Telemedicine project was used. The data obtained from the study show that the use of the Information Technology and Telemedicine tools in the care of the LCC allows to obtain savings both in economic terms and in terms of time spent, with an improvement in the results and perceived quality of care. For these reasons, this modern possibility of therapeutic approach can represent a secure resource for the sustainability of the system of treatment of this chronic pathology, worthy of further study.
\end{abstract}

\section{INTRODUCTION}

Lasting for one year, from April 2016 to April 2017, at the ASP of Cosenza, a telemedicine project took place, involving its II level Vulnology outpatient clinic.

Correspondence: Francesco Giacinto, Ambulatorio Sperimentale di Vulnologia CAPT Praia a Mare, ASP Cosenza, Località S. Stefano Praia a Mare, 87028 Cosenza, Italy.

Tel.: +39.335663809.

E-mail: drfrancesco.giacinto@gmail.com.

Key words: chronic skin wounds, wound, wound care, telemedicine.

Contributions: FG: study concept and design, study supervision and coordination, manuscript final approval; $\mathrm{CF}$ : study supervision, manuscript final approval; EG: data analysis and interpretation; $\mathrm{MG}$, DC: manuscript revising, data analysis and interpretation.

Conflict of interest: the authors declare no potential conflict of interest.

Funding: none.

Received for publication: 26 December 2017.

Accepted for publication: 11 January 2018.

This work is licensed under a Creative Commons Attribution NonCommercial 4.0 License (CC BY-NC 4.0).

(C) Copyright F. Giacinto et al., 2018

Licensee PAGEPress, Italy

Italian Journal of Wound Care 2018; 2(1):8-12

doi:10.4081/ijwc.2018.20
The objective of this study was to highlight problems and needs of the ASP in the field of home treatment of skin ulcers.

Telemedicine is a method of providing health care services, through the use of innovative technologies [...] in situations where the health professional and the patient are not in the same place.

To use this method, the secure transmission of medical data in the form of texts, sounds, images, etc. for the prevention, diagnosis, treatment and subsequent monitoring of patients is required.

This definition is specified in the National Guidelines for Telemedicine, provided by the Ministry of Health. In this document, Teleconsultation is defined as a health act, in which the doctor interacts remotely with the patient, that may give rise to the prescription of drugs or treatments; during Teleconsultation a healthcare professional who is close to the patient, can assist the doctor; the connection must allow to see and interact with the patient and must take place in real or delayed time. ${ }^{1}$

According to this concept it is therefore possible to make a remote diagnosis, with the support of films and photographs, thus defining a treatment protocol and the prescription of medications, in order to solve the problem, as well as prevention or follow up after recovery.

\section{MATERIALS AND METHODS}

In our territory we started a project of Teleconsultation and, more generally, of Telemedicine in which, with the 
use of technological devices (tablets) using an application (W. App) - supplied to us, free of charge, by pharmaceutical company C. - patients who needed medical assistance for Chronic Skin Wounds (CSW) could be followed remotely, directly to their home.

With Resolution No. 1700 of $22-9-2015,{ }^{2}$ the ASP of Cosenza, authorized us to use this method as a system for home care, to train dedicated nursing staff and to include in the project all patients affected by Chronic Skin Wounds (CSW), trying to involve also the pharmaceutical service and the evaluation nucleus for health management (which identifies the need for home care of the patients and, consequently, their insertion in the project).

In our opinion, Telemedicine services must be assimilated to any other diagnostic / therapeutic health service, as Teleconsultation does not replace the traditional health provision in the personal doctor-patient relationship, but integrates it to improve its efficacy, efficiency and appropriateness, and must comply with all the rights and obligations proper to any health act.

\section{Recruitment and evaluation of the study sample}

The recruitment phase was divided into two parts, the first regarding the patient's request, the second, its acceptance. This phase was then followed by the evaluation of the patient to be included in the sample, with a first contact through the figure of the nurse, and a final contact with the vulnology specialist. The phases can be exemplified as follows.

\section{Specific request for health care}

The patient, or the caregiver, and/or family members ask for home care because of the risk or presence of pathology, identified as CSW, by written request of the GP - who carries out the first general assessment - to the SAP (Single Access Point) of the ASP through a form called S.Va.MA. ${ }^{3}$ This form, whose acronym stands in Italian for form for multi-dimensional evaluation of adults and the elderly is a single regional tool for the evaluation of elderly not self-sufficient people asking for residential or semi-residential assistance regulated by the DPGR CA n. 36 of 21 March 2014. ${ }^{4}$

\section{Clinical assessment of care needs}

The SAP of the Health District, in Praia a Mare, receives the S.Va.M.A. and evaluates it through the MEU (Multidimensional Evaluation Unit), a multidisciplinary team composed by the Director of the Primary Care Operating Unit, responsible for it, by the Social Assistant, by the Nursing Coordinator of the ADI (Integrated Home Care Services). They identify the most appropriate interventions for the case and make up the IAP (Individual
Assistance Plan). They enter the patient's personal and anamnestic data on the W.App application on the tablet and create an electronic medical record (with a personal identification code for the patient) on which both the Vulnology specialist and the Wound Care nurses will be able to work.

\section{Evaluation of the patient and meeting with the specialist nurse}

At the same time of the creation of the electronic medical record the first meeting with the nurse is scheduled. $\mathrm{He} / \mathrm{she}$ will go to the patient's home in order to gather information, through multimedia files (photos, videos, etc.) of CSWs requiring specialistic assessment. W.App allows to identify the site of CSW on a virtual human body, inserting images of the wound itself, with the possibility of defining its contours. In this way, by calculating the wound area on consecutive pictures, it is possible to create a curve of regression and/or progression of the lesion, to get an idea of the prognosis itself. It is also possible to describe odor, characteristics of the lesion, previous medications and, more generally, signs and symptoms reported by the patient, in order to give a complete view of the situation to the specialist who will evaluate it at the same time or in a delayed one.

\section{Patient assessment, specialist contact and treatment protocol}

The specialist, after synchronizing the tablet, can perform an assessment of the wound also offline, thus starting the best treatment suitable for it and defining the amount of home accesses needed for changing/controlling the medication/treatment performed. It is also possible to prescribe wound dressings, thanks to the database containing all the products and medical devices available for the specialist, synchronized with the pharmacy (option not used during our project, given the impossibility of telematic communication with pharmaceutical services).

What we have described has been made possible thanks to the peculiarities of the software used, which has the following characteristics: i) Encrypted archive to guarantee the privacy of patients; ii) Access to the system protected by personal ID and password; iii) Possibility to send prescriptions (drugs, wound dressings and medical aids) via e-mail both to the patient and to the person in charge of the pharmacy of the district; iv) Possibility of sending a document as PDF to a consulting doctor, showing all therapies performed and prescribed, as well as the pictures of the wound and its data; v) Export of main data as Excel sheet; vi) Calculation of costs incurred for patients' care on the basis of used dressings, prescribed drugs and performed laboratory tests. 


\section{RESULTS}

The use of this application and of technologically advanced communication tools, within a precise and coordinated diagnostic and therapeutic plan for cure and care of CSWs, allowed us to achieve some important goals.

First of all we have learnt how to make savings, reducing the costs of assistance, yet managing to provide personalized and innovative care, optimizing resources, with a streamlining of the bureaucratic and management part of the assistance, finally arriving at an updating of healthcare models in wound care.

In order to better clarify our objectives and to highlight the results of this study, we show in the following tables, assistance data collected in the period October 2015-April 2016. In this period both outpatient care and the tradi- tional home care were used. Shown data are taken from the records of the services of our District.

In the year of experimentation of this new technology, April 2016-April 2017, and in particular in the study period, October 2016-April 2017, 237 patients were recruited, showing significant data on the actual savings of Health Services.

The sharp decrease in the direct specialist/patient home visits revealed after the use of tablets for telemedicine compared to those of the traditional home care model. Figures 1 and 2 show how the maximum peak of home visits decreased from a maximum of 245 performances, in February 2015/2016, to a significantly better value of 81 performances, which is the peak of the second reference period in 2016/2017. ${ }^{5}$

The second chart shows reduction of physical dis-

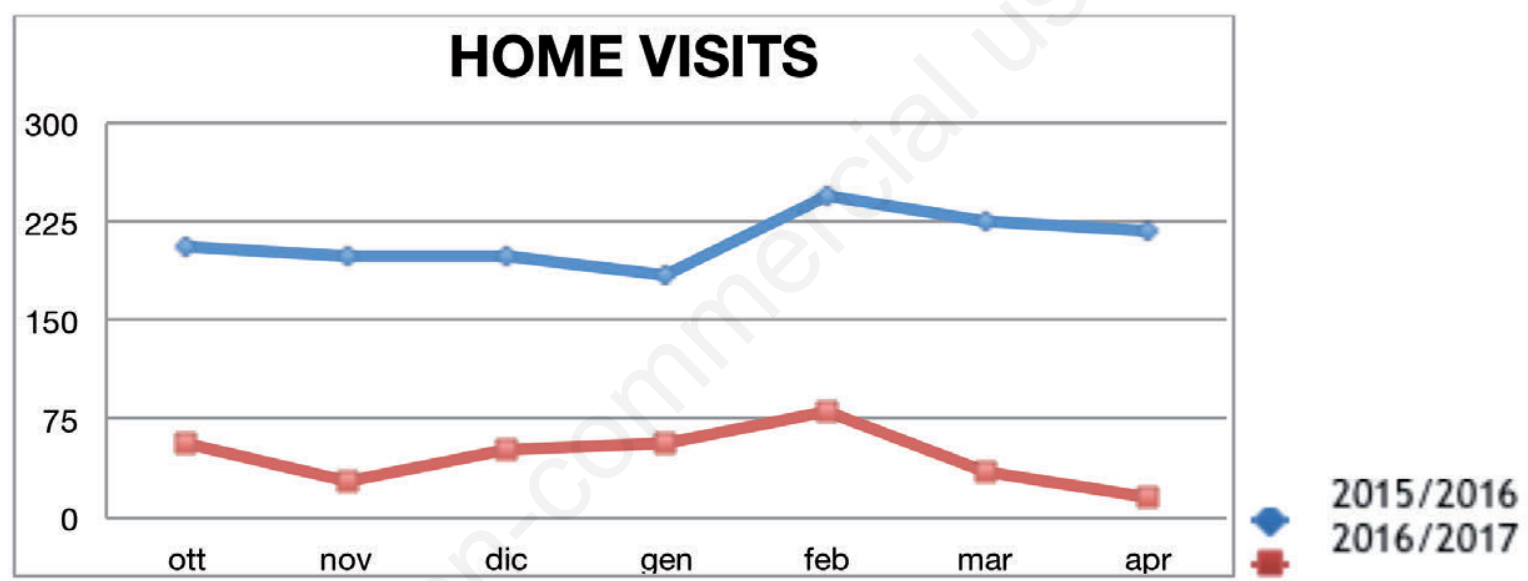

Figure 1. Comparison between home visits in the two reference periods.

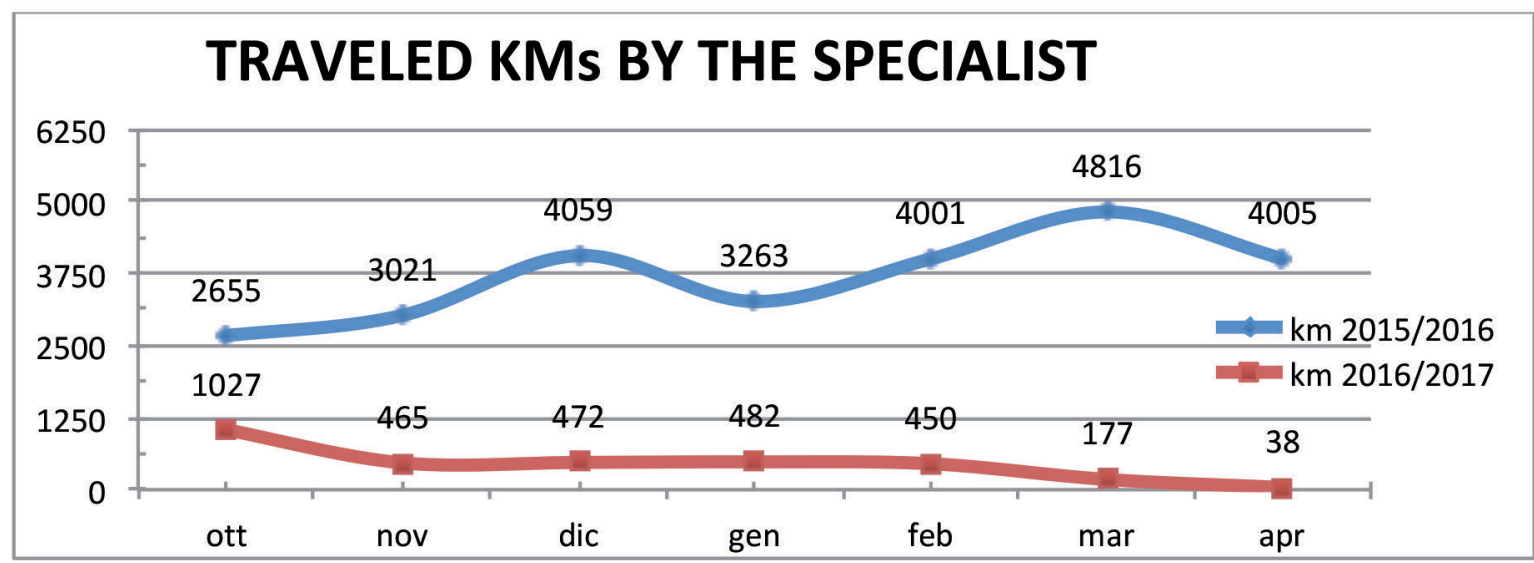

Figure 2. Comparison between $\mathrm{Km}$ traveled by the specialist in the two reference periods. 
tances between the user and the specialist, with consequent lower risk for the same and decrease of the kilometers traveled for the patient's home meeting; it , highlights the decrease of the kilometric reimbursement indemnity from a maximum of $4816 \mathrm{~km}$ traveled in the first period to a maximum of $1027 \mathrm{~km}$ in the second period. ${ }^{6}$ Figure 3 highlights the subdivision of all the assistance work, referring to the different periods. ${ }^{7}$

Comparing previously obtained data, with respect to the performances, and evaluating the actual costs sustained by National Health Service to provide this service,
Figure 4 shows both the differences, expressed in numbers, of home visits between the two periods, and the distances traveled by the specialist together with the amount of teleconsulences performed in the second period (Figure 4). Thanks to this summary figure, considering the cost data of the individual services, we can say that the health expenditure of the NHS, comparing the two periods, is reduced by $€ 40448$. This amount, reduced by the teleconference costs totaling $€ 20425$, shows an actual saving amounting to $€ 20023$, which, calculated monthly, is $€ 2860$ /month. ${ }^{8-9}$

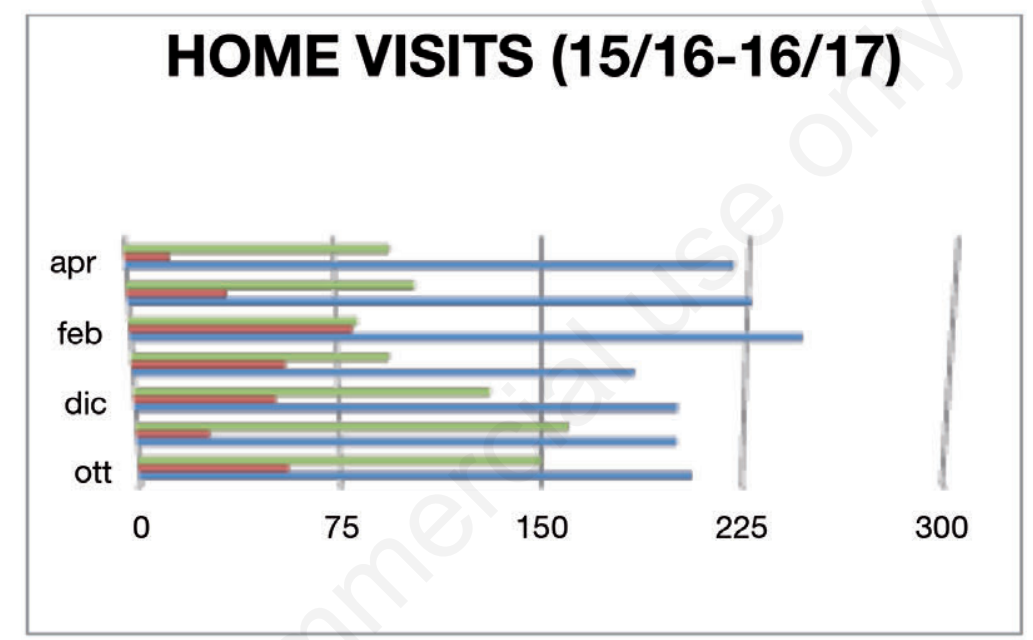

Figure 3. Comparison graphics: use of teleconsulting and comparison between number of home visits $\mathrm{n}$ in the two reference periods. The use of teleconsulence (green bar) reduced the visits to the user's home in the same year (red bar) compared to the previous year, when this method was not used yet (blue bar).

\begin{tabular}{|c|c|c|c|}
\hline & HOME VISITS & TRAVELED KMS & TELECONSULTINGS \\
\hline $\begin{array}{l}\text { October } 2015 \\
\text { April } 2016\end{array}$ & 1480 & $25820 \mathrm{~km}$ & $\mathbf{0}$ \\
\hline $\begin{array}{l}\text { October } 2016 \\
\text { April } 2017\end{array}$ & 324 & 3111 km & 817 \\
\hline DIFFERENCE & 1156 & $22709 \mathrm{~km}$ & 817 \\
\hline & COST PER HOME & TRAVELED KM COST & TELECONSULTING \\
\hline & VISIT & $0,28 \epsilon / K M$ & COST \\
\hline & $29,49 \epsilon$ & & $25 \epsilon$ \\
\hline SAVING & $34090 \epsilon$ & $6358 \epsilon$ & - \\
\hline SPENDING & - & - & $20425 \epsilon$ \\
\hline
\end{tabular}

Figure 4. Summary figure and costs comparison. 


\section{DISCUSSION}

The three cornerstones of the study can be represented in three key words: i) time: the bureaucratic process is simplified, as the user is already included in a database; ii) space: clinical assistance is brought straight to patient's bedside, at home, without the need of moving both the patient and the specialist, breaking down the physical barrier due to the vast district territory in which the project has been carried out. At the same time there is a reduction of costs regarding the mileage indemnity due to the professional; iii) cost: health services, must organize their strategic plan according to the budget provided, and need solutions that reduce costs by ensuring the same type of assistance. This guarantee is respected, using cutting-edge methods that demonstrate the lowering of costs, a reduction that in the six months of experimentation amounts to $€ 20023$, taking into account the actual teleconference costs (€ 20425).

The limit of the study is certainly the short period of reference. The small amount of available data, compared to the years 2015/2016, does not allow us to define a greater expense ratio and a better control of used resources.

\section{CONCLUSIONS}

The results obtained in this study, show that there is a way to get savings in wound car that does not necessarily excludes the quality of care: the key is in strengthening the concept of personalization of care, adapting the diagnostic and therapeutic processes to users and not vice versa.

On the other hand, it should be stressed that this system, if correctly used, provides statistical data relevant to wound care, and ensures a continuous presence of the specialist without replacing the patient's home visit, but re- inforcing the meeting with health care and best treatment protocol.

With these results, we hope that more healthcare companies can focus on the computerization of health, improving its management system. The future objective will be the assessment of the quality of provided care to the user even as perceived by himself, thus being able to confirm that the actualization of health is an excellent strategy of action.

\section{REFERENCES}

1. Chanussot-Deprez C, Contreras-Ruiz J. Telemedicine in wound care: a review. Adv Skin Wound Care 2013;26:78-82.

2. Craig J, Patterson V. Introduction to the practice of telemedicine. J Telemed Telecare 2005;11:3-9.

3. Hofmann-Wellenhof R, Salmhofer W, Binder B, et al. Feasibility and acceptance of telemedicine for wound care in patients with chronic leg ulcers. J Telemed Telecare 2006;12: 15-7.

4. Kobza L, Scheurich A. The impact of telemedicine on outcomes of chronic wounds in the home care setting. Ostomy Wound Manage 2000;46:48-53.

5. Stern A, Mitsakakis N, Paulden M, et al. Pressure ulcer multidisciplinary teams via telemedicine: a pragmatic cluster randomized stepped wedge trial in long term care. BMC Health Serv Res 2014;14:83.

6. Weinstein RS, Lopez AM, Joseph BA, et al. Telemedicine, telehealth, and mobile health applications that work: opportunities and barriers. Am J Med 2014;127:183-7.

7. Chen $\mathrm{CH}$, Young $\mathrm{TH}$, Huang $\mathrm{CH}$, et al. Patient-centered wound teleconsultation for cutaneous wounds: a feasibility study. Ann Plast Surg 2014;72:220-4.

8. Vowden K, Vowden P. A pilot study on the potential of remote support to enhance wound care for nursing-home patients. J Wound Care 2013;22:481-8.

9. Terry M, Halstead LS, O'Hare P, et al. Feasibility study of home care wound management using telemedicine. Adv Skin Wound Care 2009;22:358-64. 